\title{
Students' Understanding of Graphical Vector Addition in One and Two Dimensions
}

\author{
Umporn Wutchana ${ }^{1}$ and Narumon Emarat ${ }^{2, *}$ \\ ${ }^{1}$ Institute for Innovative Learning, Mahidol University, 999 Phuttamonthon 4 Road, Salaya, Nakhon Pathom \\ 73170, Thailand \\ ${ }^{2}$ Department of Physics, Faculty of Science, Mahidol University, 272 Rama VI Road, Ratchathewi, Bangkok \\ 10400, Thailand
}

Received: 27 December 2010 - Revised: 01 March 2011 - Accepted: 09 March 2011

\begin{abstract}
Understanding initial topics in physics (forces, fields, and kinematical quantities) requires a grasp of basic vector concepts. In this study, we intended to explore students' qualitative understanding of graphical vector addition in one and two dimensions. Using two free-response problems of a diagnostic quiz, one is one-dimensional vector addition and the other is two-dimensional vector addition. Sixty-three grade ten high-school students' responses were categorized. From the investigation, results represent that from the high-school students who already completed their vector lesson, only $10 \%$ of them provided correct answer for vector addition in one-dimension and $32 \%$ for the two-dimensional addition.
\end{abstract}

Keywords: Vectors; Vector Addition; Misunderstanding

\section{Introduction}

Vectors are component of mathematics which is an essential language for physics (Knight, 1995). Normally, high-school students face vectors as the first topic when they start learning their first physics course. Even in general university physics textbooks, vector concepts are set as the first topic. In Thailand also, for both high-school and university levels, students have to study a lesson of vectors. Basically, the vector concepts (vector magnitude, direction, addition and subtraction) are included in the introduction chapter of physics textbook. Lacking of understanding about vectors might cause surprising and serious problems when the vector concepts are embedded in almost all physics concepts (Aguirre, 1988; Aguirre \& Rankin, 1989). One also said that students' difficulty in learning physics is caused by a lack of skills and understanding about mathematics especially vectors (FloresGarcía et al., 2008). Thus, understanding vector quantity is necessary in success of learning physics. However, many physics education researchers found that students still hold misconception about vectors although they have studied it before (Knight, 1995; Nguyen \& Meltzer, 2003; Flores et al., 2004; Shaffer \& McDermott, 2005). 
In this study, we intended to explore students' qualitative understanding of graphical vector addition in one and two dimensions after being taught with a traditional lecture style. Since most physics teachers in Thailand still use traditional teaching methods, a radical change of the existing methods seems to be problematic for the teachers as a large amount of course content needs to be covered. Thus, it is interesting to know how high-school students who already completed their vector lesson with traditional class understand the concept of graphical vector addition which is the basic concept needed to understand physics. Physics instructors could get a better idea of how to set up an appropriate teaching approach to enhance their students' understanding when they know what difficulties the students have with vector addition. This study aims to investigate Thai high-school student understanding of graphical vector addition after traditional instructions.

\section{Background}

It might be deduced from previous studies that understanding of initial topics in physics requires the ability to reason about vectors (Knight, 1995; Nguyen \& Meltzer, 2003; Flores et al., 2004; Shaffer \& McDermott, 2005). Students require a good grasp of basic vector concepts to succeed in a physics course (Sheets, 1998). As the starting point, Knight (1995) surveyed students' pre-understanding of vector concepts before starting calculus-based physics course using his Vector Knowledge Test focusing on algebraic aspects of vectors. The results from his study showed that less than half of those students have sufficient skills with vectors to read the text and solve typical problems although they have studied the concept of vectors before. In the years 2000-2001, for both fall and spring semesters, Nguyen and Meltzer (2003) investigated 2,031 physics students' understanding of vector addition, magnitude, and direction for problems presented in graphical form. They administered a seven-item quiz, including free-response problems, in all introductory general physics courses at Iowa State as pre/post-test. Results showed that most of the students were unable to carry out two-dimensional vector addition after completing a physics course. Flores et al. (2004) described some of the procedural and reasoning difficulties they observed in students' use of vectors. Consistent with the work of Nguyen and Meltzer (2003), students have difficulties performing basic vector operation. They found that many students were not able to add or subtract vectors graphically after traditional instruction, and could not answer qualitative questions about vector addition and subtraction. Shaffer and McDermott (2005) investigated the ability of university students to treat velocity and acceleration as vectors in one and two dimensions. Some vector difficulties were identified among those students. Not only the introductory students but also many precollege teachers and even graduate students, vector operation difficulties were observed. Flores-Garc'ia et al. (2008) concluded from the literatures that most students have difficulties with vector addition and subtraction for both with and without physical context. After traditional instruction in introductory physics, some students still have those difficulties while a few researches have tried to improve student understanding of vector concepts (Roche, 1997; Sheets, 1998; Shaffer \& McDermott, 2005). In our study, a traditional instruction in the Thai context is investigated to see how it affects high-school students' understanding.

\section{Methodology}

We collected data from 2 classes of grade ten students, one class with 32 students and the other class with 31 students, from a medium size public school containing approximately 900 students and 48 teachers. These two classes took their first physics course with the same physics teacher. The course was taught using traditional lecture style for both classes in which the teacher always dominates the class. During the lecture, the teacher presents material to 
students and the students learn passively; listening, taking notes, and rarely asking questions. Occasionally, the teacher would ask some students to show how to solve problems in front of the class. The first chapter taught in these classes was Introduction chapter including physical quantities, SI units and vectors (focusing on magnitude, direction, addition and subtraction).

The lecture on the vector content is given two times within one week. Each lecture was given on a separate day and lasted for two hours. Graphical vector addition was taught as part of the first two-hour lecture. The graphical vector addition was first transferred to students simply by telling. Teaching by telling is mostly used for other topics in this course. The teacher told the students how to add two vectors graphically using the "tip-to-tail" strategy. This is how the teacher told the students:

To add any two vectors graphically, we start with drawing an initial vector while its magnitude and direction is preserved. Then, connect the initial vector's tip to the second vector's tail and while doing this the magnitude and direction of the second vector should also be preserved. Finally, a new vector is formed by dragging from the initial vector's tail to the second vector's tip. This new created vector is called a resultant vector.

After this, the teacher showed the whole class how to add vectors graphically in one dimension and the students copied everything into their own notes. Then, all students were assigned to work with some examples. One or two students were then asked to give the solution in front of the class. Right here, some student mistakes would be corrected and students were allowed to ask any question that they might have. After this, graphical vector addition in two dimensions was presented to the students with the same teaching strategy. After completion of the lecture, some vector addition problems were assigned as student homework.

The two classes finished their first chapter nearly at the same time and faced all the same activities including in-class examples and homework problems. Before starting the next chapter, a diagnostic quiz measuring students' understanding of vector direction, magnitude and addition presented in a graphical form was administered to the students. Students in both classes did not learn about vector before and since vector content is not what we learn or face in daily life, especially for graphical vector addition and subtraction, it is acceptable that the students had no prior knowledge about this topic. Therefore, pre-test is considered not essential in this study. The diagnostic quiz was translated from the work of Nguyen and Meltzer (2003). It is a seven-item quiz, including problem 1: vector magnitude, problem 2: vector direction, problem 3: qualitative vector addition, problem 4: one-dimensional vector addition, problem 5: two-dimensional vector addition, problem 6: two-dimensional vector subtraction, and problem 7: comparison of resultant magnitude. Students were asked to give a free response for problems 1,2, 4, 5 and 6 and to select multiple options from a list for problems 3 and 7. Students spent 20 minutes to complete the quiz and their results were not counted as a course grade.

To understand various algorithms that students used for adding vectors in one and two dimension, two free-response problems (problems 4 and 5) of the diagnostic quiz presented in figure 1 were analyzed. Only these two problems measure student graphical vector addition while the other problems focus on magnitude, direction and subtraction of vectors. Although problem 3 asks students to add vectors qualitatively, it is set as a multiple choice format question. The students just have to select a vector answer that has the same direction as the resultant vector. Therefore, the answers may not reveal students' idea of graphical vector addition. Problem 3 was then omitted in this paper. Then, the student responses were categorized in order to explore their qualitative understanding. The students were not asked to 
provide any explanation for their answers but their drawings can reveal how they figure out their answers.

\#4: In the space to the right, drew $\vec{R}$ where $\vec{R}=\vec{A}+\vec{B}$. Clearly label it as the vector $\vec{R}$.

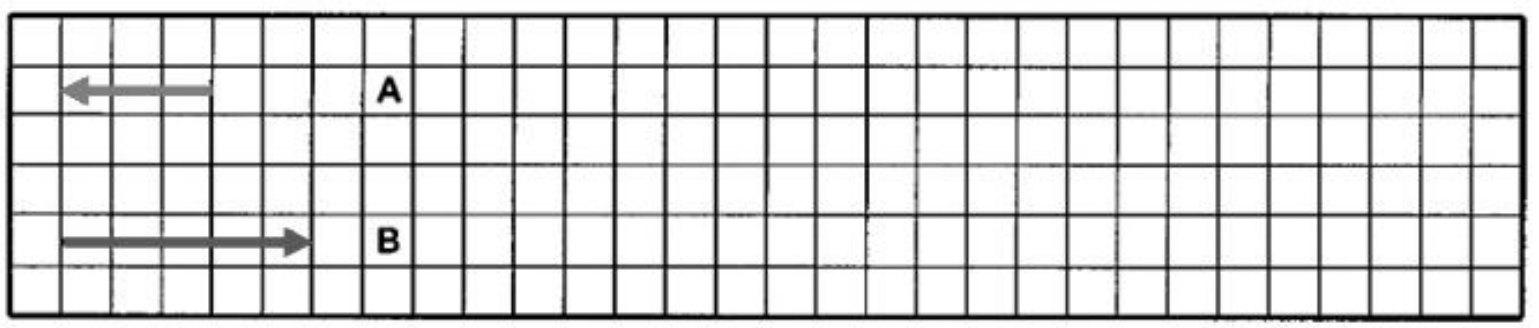

\#5: In the figure below there are two vectors $\vec{A}$ and $\vec{B}$. Draw a vector $\vec{R}$ that is the sum of the two, (i.e., $\vec{R}=\vec{A}+\vec{B}$ ). Clearly label the resultant vector as $\vec{R}$.

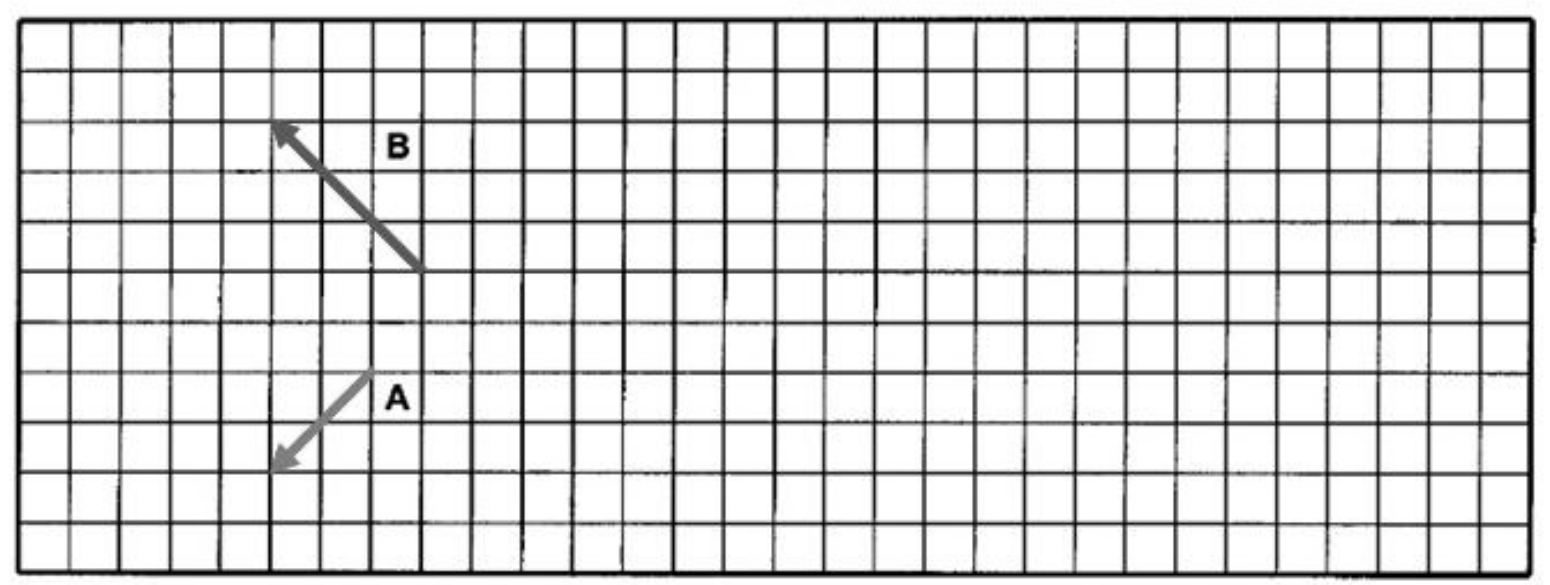

Figure 1. Two free-response problems of the diagnostic quiz probing students' understanding of one and two-dimensional vector addition (Nguyen \& Meltzer, 2003)

\section{Results and Discussion}

\section{One-dimensional vector addition}

Table 1. Categories of students' understanding of graphical vector addition in one dimension

\begin{tabular}{clc}
\hline Category & \multicolumn{1}{c}{ Description } & Students (\%) \\
\hline (a) & Correct answer & 10 \\
(b) & Missing direction of $\vec{R}$ & 3 \\
(c) & Various ways to attach the two & \\
& vectors but gives no $\vec{R}$ & 49 \\
(d) & Connecting original vectors from tail & \\
& to tail & 11 \\
(e) & Making various triangles & 13 \\
Other & Other answers & 3 \\
No answer & No answer & 11 \\
\hline
\end{tabular}


Table 1 presents the category of the 63 grade ten students' responses to problem 4 which was used to classify students' understanding of one-dimensional vector addition. The students' responses were categorized into 7 categories labeled as (a), (b), (c), (d), (e), Other, and No answer. The category description and percentages of students giving those answers are presented. There are only $10 \%$ of the students provided correct answers to problem $4.3 \%$ of them drew the resultant vector $\vec{R}$ without its direction. Surprisingly, students added the one-dimensional vectors following incorrect algorithms. Almost half of the students attached the two vectors, in one dimension, in different ways and gave no resultant vector $\vec{R} .11 \%$ of them connected the two original vectors as shown in the problem from tail to tail and the resultant vector appeared two dimensions. 13\% re-oriented the vectors and made various triangles while $11 \%$ gave no answer. Examples of the students' drawings and answers in each category are presented in Figure 2.

(a)

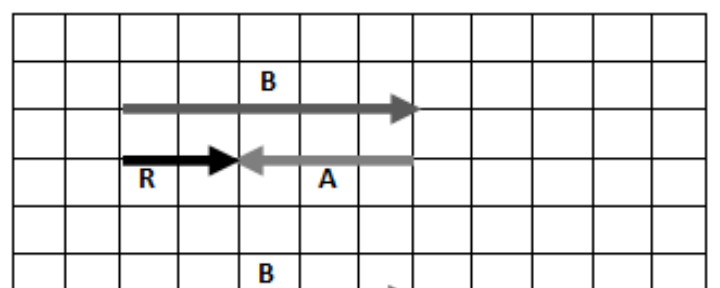

(c)

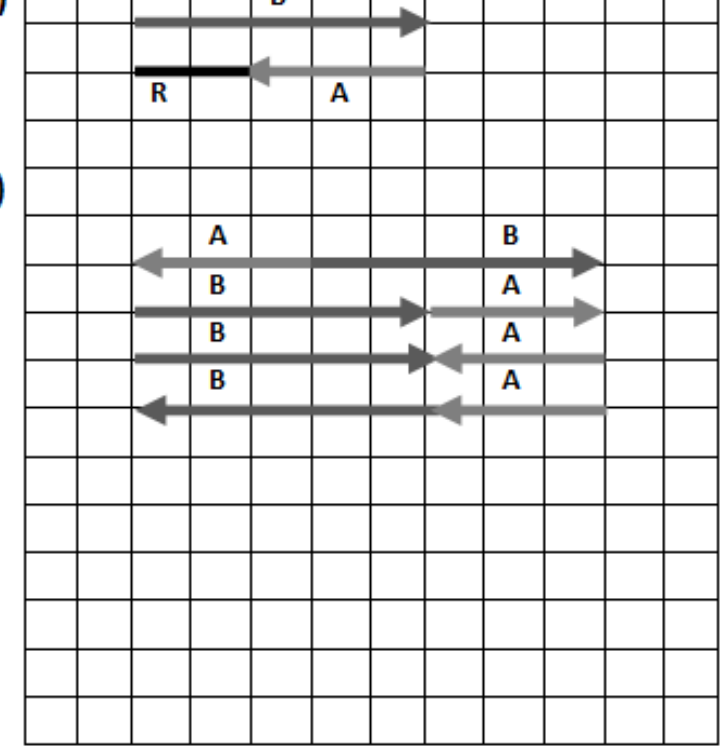

(d)

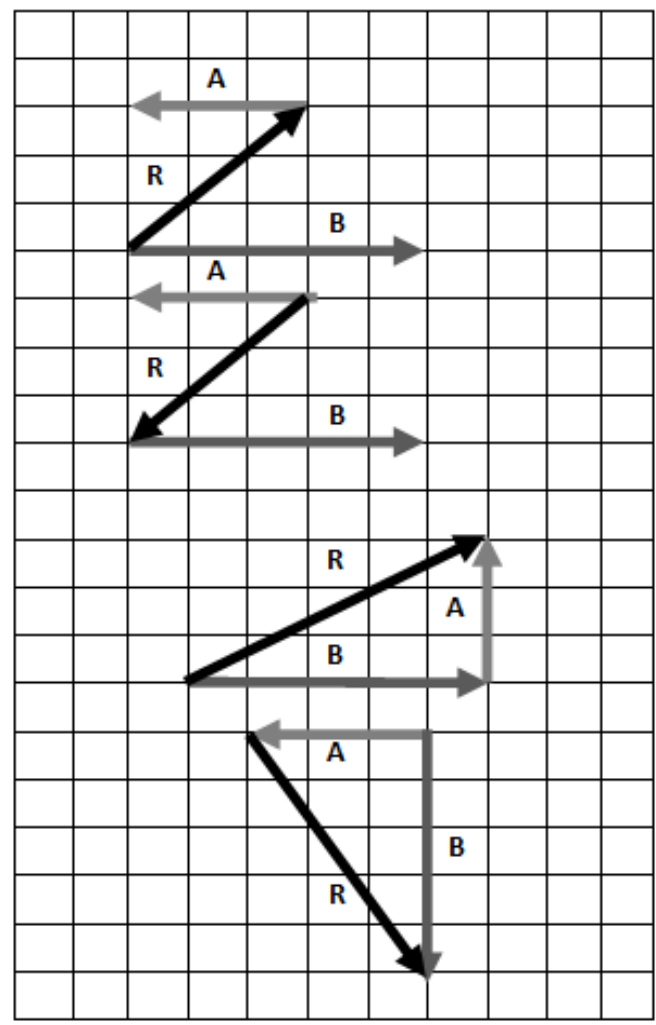

Figure 2. Students' responses to graphical vector addition in one dimension: (a) provide correct answer; (b) miss direction of $\vec{R}$; (c) attach the two vectors with no $\vec{R}$; (d) connect the original vectors from tail to tail; (e) re-orient the vectors and create triangles.

The students' misunderstanding in categories (c), (d) and (e) is consistent with the finding of Nguyen and Meltzer (2003). In our case, we also found two-head arrows with eight boxes long for category (c). There are various kinds of arrows connection as shown in figure 2(c). For category (b), the students' answer seems to be correct but miss the direction of $\vec{R}$. Possible reasoning is that the students forgot to put the direction of $\vec{R}$. For category (d), students did not make an effort to re-draw vectors $\vec{A}$ and $\vec{B}$ to attach them together. They simply drew a resultant vector $\vec{R}$ as a connection between the two original vectors shown in the problem. For category (e), it is quite a surprise that the students re-oriented the vectors. 
Student's answers in categories (d) and (e) show that they did not realize that they were adding vectors in one dimension and so the resultant vector should be one dimensional.

\section{Two-dimensional vector addition}

Table 2. Categories of students' understanding of graphical vector addition in two dimensions

\begin{tabular}{clc}
\hline Category & \multicolumn{1}{c}{ Description } & Students (\%) \\
\hline (a) & Correct answer & 32 \\
(b) & Missing direction of $\vec{R}$ & 10 \\
(c) & Wrong direction of $\vec{R}$ & 19 \\
(d) & Connection of the two vector tips & 5 \\
(e) & Making various triangles & 14 \\
(f) & Connecting original vectors from tip & \\
& to tail or tail to tip & 6 \\
Other & Other answers & 3 \\
No answer & No answer & 11 \\
\hline
\end{tabular}

Table 2 presents 8 categories of the students' responses to problem 5. This problem was used to classify students' understanding of two-dimensional vector addition. The categories are labeled as (a), (b), (c), (d), (e), (f), Other, and No answer. The description and percentage of students in each category are presented. The students' graphical answers in each category are presented in Figure 3.

Higher than those of problem 4 , there are $32 \%$ of the students provided the correct answer to problem 5 . $10 \%$ of them drew $\vec{R}$ without its direction and $19 \%$ drew $\vec{R}$ with a wrong direction. These represent that the students did not really understand how to perform two-dimensional vector addition graphically. Categories (a), (b) and (c) show us that the students could re-draw and connect the two vectors correctly but gave the wrong or even missed the direction of the resultant vector. $5 \%$ of the students connected the two vector tips together after the vector tails were attached as presented in figure $3(\mathrm{~d}) .14 \%$ of them reoriented and/or re-sized the vectors and made various triangles. $6 \%$ of the students connected the two original vectors from tip to tail or tail to tip without re-drawing and attaching the two vectors together while $11 \%$ gave no answer.

From the 5 categories ((b) to (f)) of students' misunderstanding of two-dimensional vector addition, only category (d), connecting the two vector tips together, is consistent with the finding of Nguyen and Meltzer (2003). Differ from this study, we found that most of the students provided answers either with missing or wrong direction of $\vec{R}$. Some students surprisingly made triangles with no understanding while others only made a connection between the original vectors presented in the problem, as shown in figures 3(e) and 3(f), respectively. 
(a)

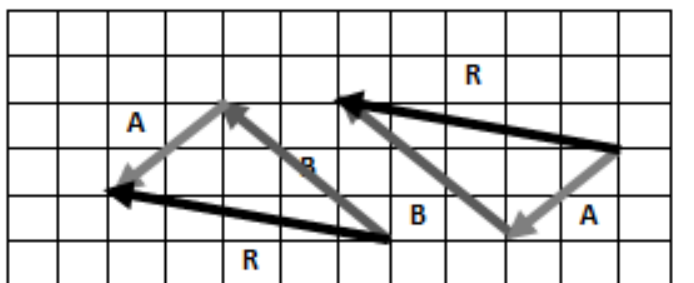

(b)

(c)

(d)
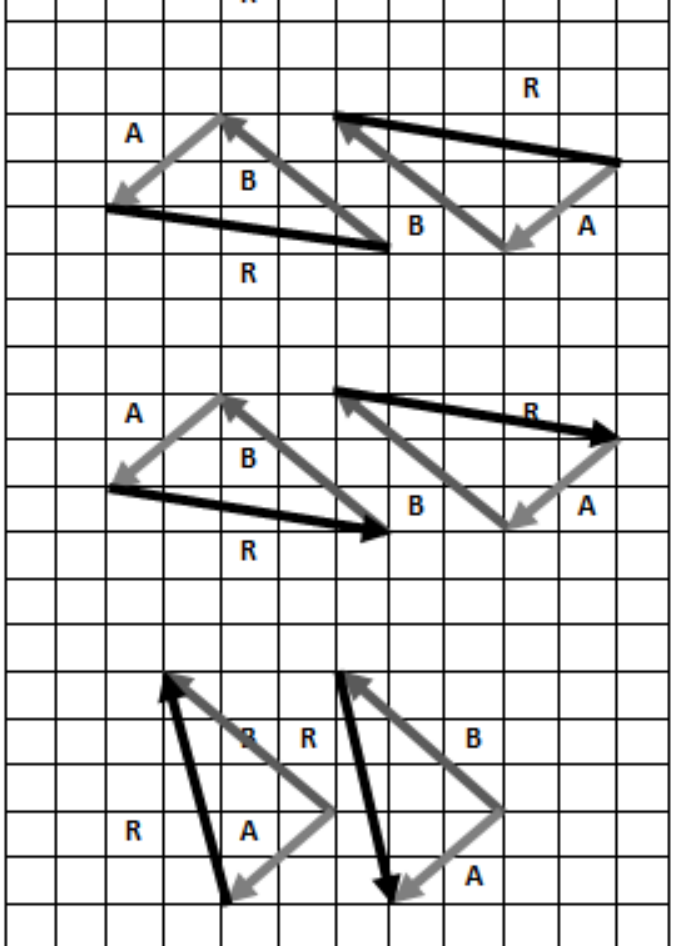

(e)

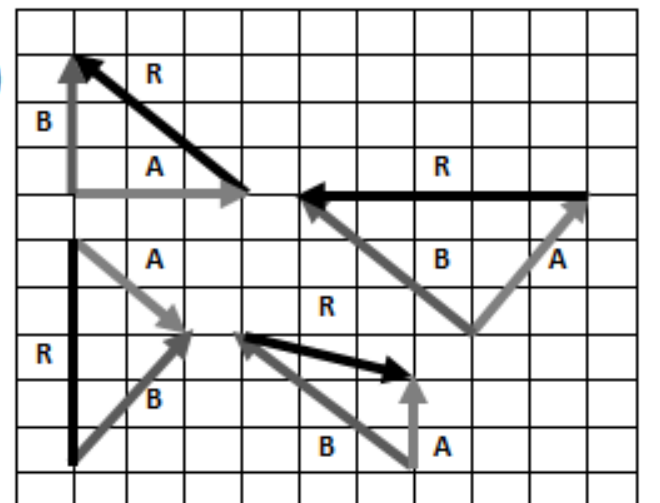

(f)

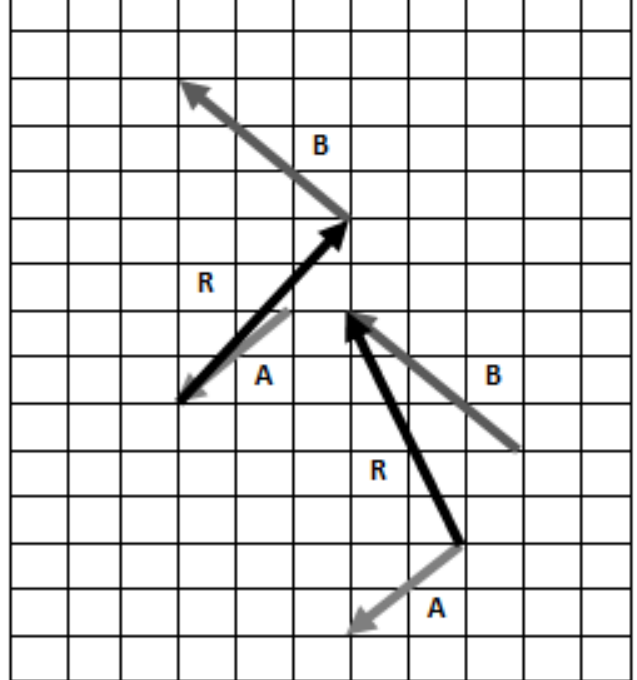

Figure 3. Students' responses to graphical vector addition in two dimensions: (a) provide correct answer; (b) miss direction of $\vec{R}$; (c) provide wrong direction of $\vec{R}$; (d) connect tips of the two vectors; (e) re-orient the vectors and create various triangles; (f) connect the original two vectors from tip to tail or tail to tip.

It might be deduced from our findings that there are six categories of student misunderstanding about graphical vector addition in one and two dimensions. The misunderstanding comprises (1) missing the direction of $\vec{R},(2)$ giving a wrong direction of $\vec{R}$, (3) attaching two vectors in various ways and not giving $\vec{R}$, (4) connecting two original vectors without making an attachment, (5) making various triangles, and (6) connecting between two vector tips.

For category (1), when looking at the student responses in figures 2(b) and 3(b) we can see that the students already attached the two vectors correctly and gave the correct magnitude of the resultant vectors. It is possible that the students simply forgot putting the direction of $\vec{R}$ or they might not be aware of all the vector properties. The percentage of students in this category was found more in the two-dimensional vector addition problem. To avoid this misunderstanding, physics instructors should emphasize that all vector quantities comprise magnitude and direction and should spend some times to check if students understand about this point during classes.

Wrong directions of $\vec{R}$ in category (2) occurred in the two-dimensional vector addition (see category (c) in table 2 and figure 3(c)). The results show that students made the attachment between the two vectors correctly, using a "tip-to-tail" strategy, but finally they 
gave the wrong direction of $\vec{R}$. By looking at figure 3(c), one possible explanation is that the students tried to make the arrow of $\vec{R}$ so that the formed triangle is "flowing" in one direction. They might not remember when the teacher told them how to draw a resultant vector. The only thing they could remember seems to be the "tip-to-tail" connection.

For category (3), a large number of students attached two one-dimensional vectors following incorrect algorithms (see figure 3(c)). They added the vectors by attaching them on the same horizontal line and in various ways: tip to tip; tail to tail and; tip to tail. Some students did not preserve the direction of those vectors and, more importantly, all of them gave no resultant vectors. It is possible that when they attached two vectors on the same line the students did not know where to draw the resultant vector $\vec{R}$ or they might think that the attachments themselves were already the resultant vectors. The students in this category did not know that the one-dimensional vectors shown in the quiz can be attached on different lines so that a resultant vector can be drawn more obviously. Perhaps the instructors should show how to do this to students through many examples.

In category (4), students connected two original vectors presented in the problem from tail to tail (see figure 2(d)) and tip to tail or tail to tip (see figure 3(f)). No attempts were made in order to attach the two vectors together. These students might not know or were not assured that the vectors can be copied and moved to somewhere else while their magnitude and direction are preserved. The one thing they were certain of is that the resultant vector is formed by drawing a connection between the two given vectors. However, some could not remember exactly which direction their resultant vectors should be drawn; tip to tail or tail to tip.

Making various triangles in category (5), as shown in figures 2(e) and 3(e), is different from students' misunderstanding found in category (4). These students knew that vectors can be moved but they were not aware that the magnitude and directions have to be preserved.

The findings of categories (3), (4) and (5) suggest that before students learn how to add vectors in one and two dimensions, instructors should assure the students that vectors can be moved and also ensure that they must preserve vectors' magnitude and direction while moving them. An exercise or homework on moving vectors might be needed.

For the last category, connection of two vector tips is found only in the two-dimensional vector addition (see figure 3(d)). We can see that the students knew how to move those vectors and preserve their magnitudes and directions. The major problem for these students is that they did not know the correct way to attach the two vectors. They simply attached the vector tails together and then made the connection between the vector tips to form a resultant vector. Although a small number of students in this category are found, instructors should still be aware of this misunderstanding when teaching two-dimensional addition.

From these findings, we see that after a traditional teaching on graphical vector addition, some students ignored the direction of $\vec{R}$. They did not place an arrow on the line. Many students added the vectors by creating a wrong triangle. Most of them thought that adding two vectors meant just attaching them to each other without knowing how to do it properly. Others did not understand that vectors can be moved to make a connection. These are consistent with what physics teachers expected; students will find difficulty based on their experience of how they worked in class (Poynter \& Tall, 2005). Our findings here are also consistent with the previous work that students still hold misunderstanding about vector addition after traditional teaching (Knight, 1995; Nguyen \& Meltzer, 2003; Flores et al., 2004). 


\section{Conclusion}

Consistent with the previous work, students still hold misunderstanding about vector addition after a traditional teaching. There is a variety of student misunderstanding of graphical vector addition found in our study. Student difficulties were observed when we asked the students to add vectors graphically without any physical context. The students did not grasp the important concept about the vector direction and how a vector may be moved while its magnitude and direction are preserved. Many students are still confused about the "tip-to-tail" strategy. These might cause future problems to their physics study. Student might be unable to use vectors in solving problems about kinematical quantities, forces or fields. Students might fail to add physical forces in any situation to find a net force.

Our investigation suggests that when teaching about graphical vector addition in one and two dimensions, there are four important points that the instructors should check to make sure that their students understand: 1) students realize that vector quantities comprise of both magnitude and direction; 2) students understand that a vector can be moved and while moving a vector, its magnitude and direction must be preserved; 3) students know that when adding two vectors, one vector's tip must be attached to the other vector's tail and; 4) students are clear that the direction of the resultant vector points from the tail of the initial vector to the tip of the second vector.

It is necessary to probe students' understanding of vector concepts even after the lesson. Physics instructors should make sure that students are able to carry out vector addition graphically in one and two dimensions after completing the study. A careful instruction on vectors is needed. Active learning and teaching (Roche, 1997; Sheets, 1998; Shaffer \& McDermott, 2005) are as much promise for helping students significantly improve their conceptual understanding. When accompanied with considering those four critical points the instructors could help students get higher gains on graphical vector addition in one and two dimensions. Computer based teaching is also one interesting choice which can maximize the efficiency of a teaching and learning process and increase the standards of students' achievement (Çataloğlu, 2006; Tsegaye et al., 2010). Without clearing up student misunderstanding of vector concepts, we cannot expect that students will correctly apply vectors in many basic and advanced physics topics.

\section{Acknowledgements}

The authors would like to thank all the member of the Physics Education Network of Thailand (PENThai) for many valuable discussions and suggestion. We also give thanks to the Institute for Innovative Learning, Mahidol University, the Thailand Center of Excellence in Physics (ThEP) and the Institute for the Promotion of Teaching Science and Technology (IPST) for providing in parts the support to this study.

\section{References}

Aguirre, J.M. (1988). Student preconceptions about vector kinematics. The Physics Teacher, 26(4), 212-216.

Aguirre, J.M. \& Rankin, G. (1989). College students' conceptions about vector kinematics. Physics Education, 24(5), 290-294.

Çataloğlu, E. (2006). Open source software in teaching physics: A case study on vector algebra and visual representations. The Turkish Online Journal of Educational Technology, 5(1), 68-74. 
Flores, S., Kanim, S.E. \& Kautz, C.H. (2004). Student use of vectors in introductory mechanics. American Journal of Physics, 72(4), 460-468.

Flores-García, S., Alfaro-Avena, L.L. \& Dena-Ornelas, O. (2008). Students' understanding of vectors in the context of forces. Revista Mexicana de Física E, 54(1), 7-14.

Knight, R.D. (1995). The vector knowledge of beginning physics students. The Physics Teacher, 33(2), 74-77.

Nguyen, N. \& Meltzer, D. E. (2003). Initial understanding of vector concepts among students in introductory physics course. American Journal of Physics, 71(6), 630-638.

Poynter, A. \& Tall, D. (2005). What do mathematics and physics teachers think that students will find difficult? A challenge to an accepted practices of teaching. Proceedings of the Sixth British Congress of Mathematics Education, University of Warwick, UK, 2004, p. 128-135.

Roche, J. (1997). Introducing vectors. Physics Education, 32(5), 339-345.

Shaffer, P.S. \& McDermott, L.C. (2005). A research-based approach to improving student understanding of the vector nature of kinematical concepts. American Journal of Physics, 73(10), 921-931.

Sheets, H.D. (1998). Communicating with vectors. The Physics Teacher, 33(2), 74-77.

Tsegaye, K., Baylie, D. \& Dejne, S. (2010). Computer based teaching aid for basic vector operations in higher institution Physics. Latin American Journal of Physics Education, $4(1), 3-6$. 